\title{
Automated Image Processing to Quantify Cell Migration
}

\author{
Minmin Shen ${ }^{1}$, Bastian Zimmer ${ }^{2}$, Marcel Leist ${ }^{2}$, Dorit Merhof $^{1}$ \\ ${ }^{1}$ Interdiscipinary Center for Interative Data Analysis, Modelling and Visual \\ Exploration (INCIDE), University of Konstanz ${ }^{2}$ The Doerenkamp-Zbinden Chair of \\ in-vitro Toxicology and Biomedicine, University of Konstanz \\ minmin.shen@uni-konstanz.de
}

\begin{abstract}
Methods to evaluate migration capacity of stem cells and the inhibition by chemicals are important for biomedical research. Here, we established an automated image processing framework to quantify migration of human neural crest (NC) cells into an initially empty, circular region of interest (ROI). The ROI is partially filled during the experiment by migrating cells. Based on an image captured only once at the end of the biological experiment, the framework identifies the initial ROI. The identification worked also, when the distribution of surrounding cells showed large heterogeneity. After segmentation, the number of migrated cells was identified. The image processing framework was capable of efficiently quantifying chemical effects on cell migration.
\end{abstract}

\section{Introduction}

Safety assessment of chemicals with new stem cell-based in vitro methods requires not only new biological test systems, but also technical and computer science solutions that allow for high throughput and for unbiased observerindependent data analysis. Manual scoring and manipulation of cells requires intensive, time-consuming training of operators and easily introduces bias into the data. This is particularly important for image-based methods that require quantification and classification of cells in time and/or space.

For detection of environmental toxicants that may adversely affect human development, a screening strategy based on the use of stem cells has been worked out [1]. Within this approach, a test system evaluating the migration capacity of human neural crest (NC) cells has been established.

In a new, technically optimized version of the assay (Fig. 1), cells are seeded onto the culture plates, with stoppers inserted to block cell adhesion in circular areas within a cell culture dish. After removal of the stopper, a defined cellfree area is created (the ROI). The cells are then incubated for $48 \mathrm{~h}$ with cell culture medium either containing no test chemicals, or containing different potential neurodevelopmental toxicants (C1-C5). At the end of the experiment, the cells are stained, and images of the culture dish are taken to determine how many cells migrated into the ROI. Different manual and semiautomatic scoring methods have been used in the past. For instance, the area where the stopper 
had been placed may be defined mechanically, on the basis of the recorded $x, y$ coordinates. However, this requires additional manipulation steps, and a second imaging step. Moreover, the inaccuracy of positioning and of relocating the coordinates (e.g. $0.1 \mathrm{~mm}$ ) often introduces noise into the data. Therefore, a new method is required which automatically identifies the ROI after a single imaging step at the end of the experiment, and then scores the number of migrated cells operator-independently.

To quantify cell migration, it is required to localize the ROI. However, it would be difficult to determine its exact location before acquiring cell images by manual operations, e.g. putting stamps inside or under the cell cultures. And a small mismatch would cause rather low precision due to the small size of a stopper (its diameter is $2 \mathrm{~mm}$ ). For this reason, the location of the ROI needs to be estimated using automated image processing approaches.

In this paper, an image processing framework is presented for automated cell migration analysis in response to this need. The acquisition of image data and the image processing framework are elaborated in Section 2. The results are shown in Section 3 to demonstrate the validity of the proposed framework.

\section{Material and methods}

\subsection{Experimental setup}

Oris plates (Platypus technologies, Madison, WI) were used to perform migration experiments into an area initially generated by a stopper. The cell source were human-embryonic stem cells derived NC cells [1]. They were stained with two fluorescent dyes and imaged on an automated inverted microscope, equipped with a $4 \times$ lens. The individual images from a culture are stitched together to a large image file by proprietary microscope software (Cellomics Array Scan VTI, Thermo Fisher) to allow a full overview of the ROI and its surrounding area (Fig. 2).

(a)

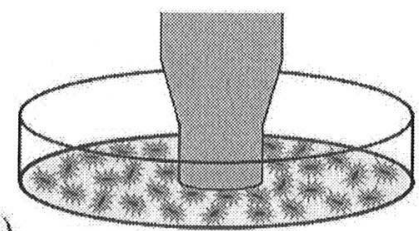

(b)

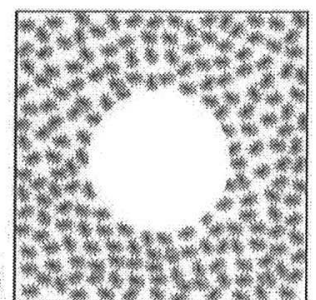

(c)

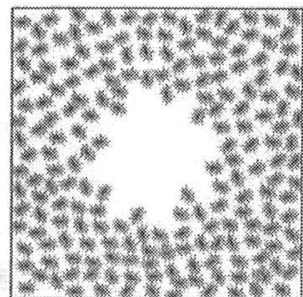

Fig. 1. Principle of the "non invasive" migration assay of NC (MINC): (a) insert stoppers and seed cells onto cell culture plate (side view), (b) remove stoppers to create detection zone (top view), (c) allow cells to migrate into detection zone (top view). 


\subsection{Image data}

Each acquired composite image of a culture dish consists of two color channels: a blue-fluorescent channel indicating nuclei (stained with H-33342) and a greenfluorescent channel indicating the total cell area (cytoplasmic stain through use of calcein-AM).

\subsection{Estimation of ROI}

The initial step to quantify cell migration is to detect the circular ROI. Due to the aforementioned difficulties to manually determine the coordinates of the stopper before acquiring cell images, an automated image processing method to estimate the ROI in a cell image is required. To estimate the circle, we select the green channel to avoid noise observed in the blue channel.

The segmentation of the central region (i.e. the area that is not populated by cells) is performed in polar coordinates, where the horizontal axis corresponds to the angle axis, and the vertical axis corresponds to the radius axis, respectively. As an example, the cell image (Fig. 3a) is first transformed to polar coordinates using the center of the whole image as the pole (Fig. 3b). Usage of the polar image improves the segmentation result over pure smoothing and thresholding the cartesian image [2]. After applying morphological operations, i.e. dilation and flood-fill operations, a binary mask is generated (Fig. 3c). It is transformed back to Cartesian coordinates and only the segment with the largest area is selected (dark region in Fig. 3d). The initial estimate of the origin of the circle is computed as the center of gravity of this segment, and the estimated ROI is outlined (Fig. 3e).

However, the initial estimate may not be reasonable for some cell images in which the cells are not evenly distributed. A "hole" is observed in these images, as shown at the bottom-right corner in Fig. 3a. Looking back at the segmentation result in Polar coordinates (Fig. 3c), the protruding part of the dark region is connected to the upper margin, corresponding to the "hole" in Cartesian coordinates. Ideally, the dark region will be a rectangle if its counterpart in Cartesian coordinats is a regular circle and the origin of the circle is set as the pole for Cartesian-to-Polar coordinates transformation.

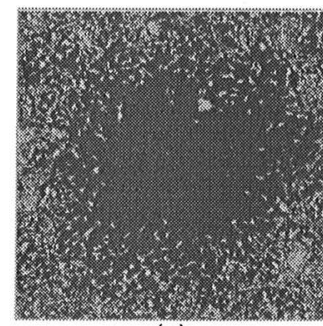

(a)

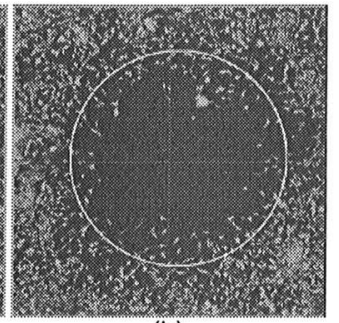

(b)

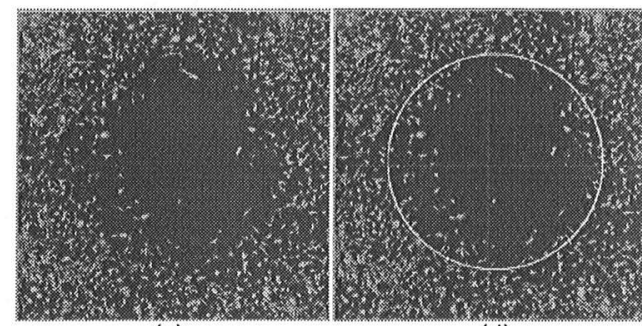

(c)

(d)

Fig. 2. Examples of dual channel microscopy images (a,c) used for identification of the ROI (b,d). 
Based on this observation, the optimal estimation of the origin $\left(x_{o p t}, y_{\text {opt }}\right)$ is a coordinate that, if it is set as the pole, the dark region in the polar image approximates a rectangle. To measure how well the dark region in the polar image approximates a rectangle, the variance of the $y$-coordinate value of its upper outline is computed. The smaller the variance, the more the dark region approximates a rectangle. $\left(x_{o p t}, y_{o p t}\right)$ is searched within a window around the initial estimate, and the one with the smallest variance is found and kept as the optimized result (Fig. 3f).

\subsection{Cell segmentation and quantitative analysis}

Once the ROI is determined, segmentation of individual cells is applied in this region. We use several KNIME (The Konstanz Information Miner [3]) nodes including Voronoi segmentation [4] for cell segmentation.

There are numerous segmentation algorithms for cell segmentation. The Voronoi-based algorithm is used in this paper due to its robustness to noise. Based on a metric defined in the image plane and based on seed points, seed-to-pixel distances are calculated and each pixel is assigned to the closest seed under that metric [4]. The nuclei images are segmented using the local maxima of each segment as the seed, and the cytoplasm images are segmented using the nuclei of each cell as the seed.

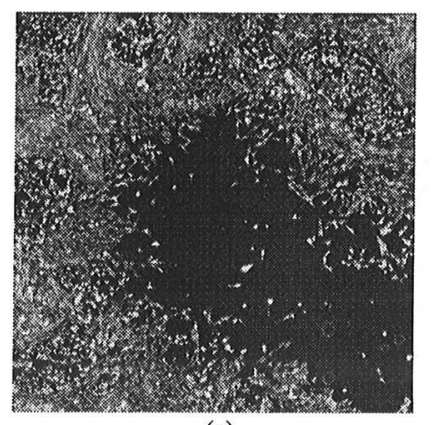

(a)

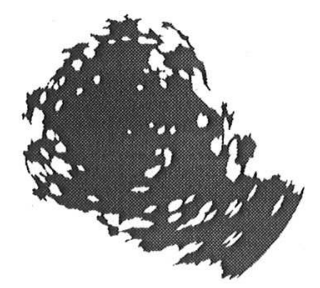

(d)

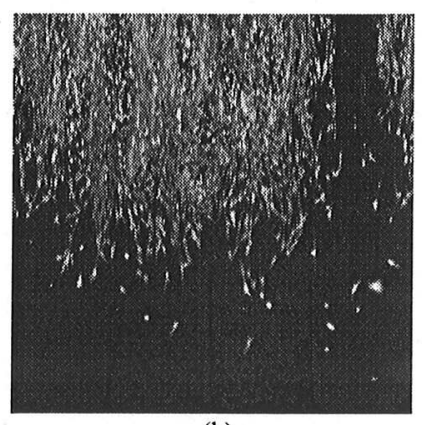

(b)

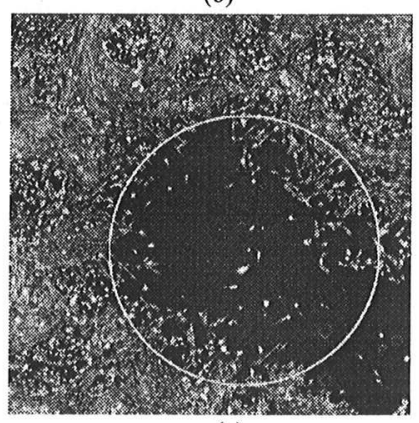

(e)

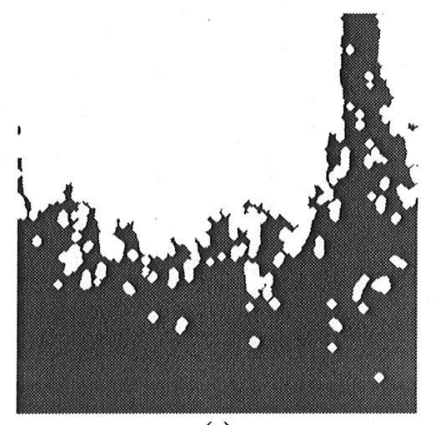

(c)

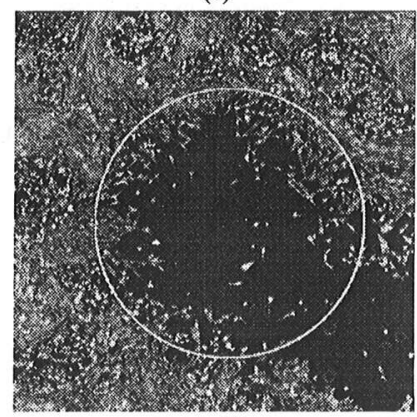

(f)

Fig. 3. An example image (green channel) with unevenly distributed cells (a) and its polar image (b). Segmentation result with morphological operations (c) and the largest segment transformed to Cartesian coordinates (d). Initial estimation of ROI (e). Optimized estimation (f). 
The number of cells within the ROI is calculated based on the Voronoi segmentation result. As there are outliers in both channels (noise in blue channel and cell debris in green channel), the cytoplasm image and nuclei image are converted to binary images using global threshoding, and are then multiplied with each other to exclude the aforementioned outliers. The multiplication results in an image with noise and cell debris excluded, and the number of cells is calculated based on this image.

\section{Results and discussion}

The proposed image processing framework was applied to 68 microscopy images of human NC cells, including both untreated cells (28 images) and cell populations treated with five chemicals (C1-C5), for each of which eight images were obtained.

In order to evaluate the performance of the proposed algorithm to estimate the location of the ROI, the origin of the circle was manually located by three human raters. Each human rater manually positioned a circle on the cell image using computer painting tools. The means and standard deviations of the Euclidean distance between the origins of circles of human raters vs. the results of the proposed algorithm (green), and between human raters only (black), are illustrated in Fig. 4a, on all six experimental groups of cell images. The mean and standard deviation of the Euclidean distance averaged across all cell images is $18.8 \pm 12.6$ pixels for human raters vs. the proposed approach (mean \pm std deviation), and $19.5 \pm 12.2$ pixels between different human raters. The results show that the estimation by the proposed approach is comparably accurate as human raters, which proves the efficacy of the proposed algorithm in estimating the ROI. The algorithm is also compared to the result of circle detection based

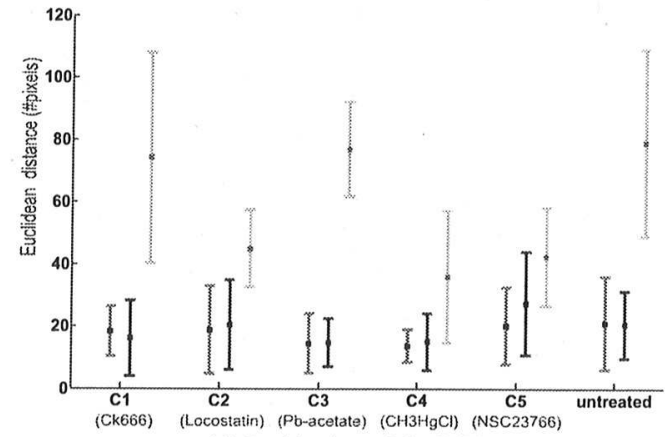

(a) Positioning of the ROI

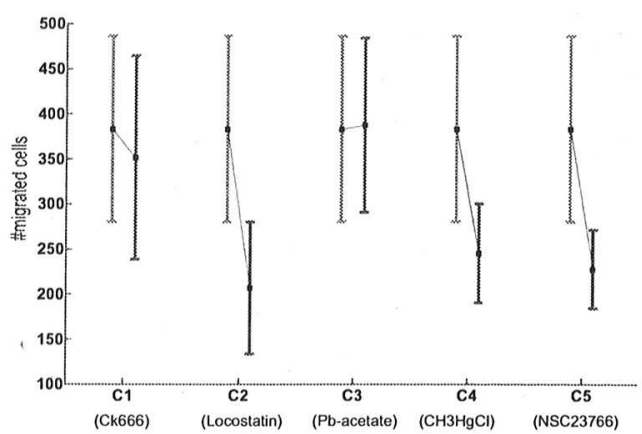

(b) Quantification of the number of cells in ROI

Fig. 4. Results for cell populations treated with five different chemicals (C1-C5). (a) Means and standard deviations of the Euclidean distance between the origins of circles of human raters vs. the results of the proposed algorithm (green), the origins of circles of human raters vs. a standard circular Hough transformation (pink), and between human raters only (black). (b) Means and standard deviations of numbers of untreated cells (red) and cells treated with different chemicals, respectively (blue). 
on the standard circular Hough transformation (Fig. 4a, pink). It can be clearly seen that the proposed circle detection outperforms the standard circular Hough transformation, which has a much higher estimation error (64.8 \pm 30.6$)$. Some example results of the estimated ROI are provided in Fig. 2.

In a next step, the accuracy of the cell counts was examined by biologists. For this purpose, the human raters manually counted the number of cells and observed the automated segmentation results of the proposed algorithm to determine the false-positives. The percentage of false-positives was within the range of $1.56 \%$ to $9.76 \%$. The results show that the segmentation algorithm tends to oversegment the cells, but according to the biologists this percentage of falsepositives is acceptable and in the range of the manual counting variation. A small trend towards oversegmentation is well acceptable to the biologists as they care more about the relative change of the number of cells after treatment with different chemicals.

Fianlly, the cell counts are used in order to assess the effect of chemicals on cell migration. For this purpose, the means and standard deviations of numbers of untreated cells and cells treated with five chemicals are shown in red and blue, respectively (Fig. 4b). For example, the number of cells decreases significantly after treatment with Locostatin (C2), which indicates that this chemical affects cell migration. In contrast, the cell number does not change significantly after treatment with $\mathrm{Pb}$-acetate (C3), which indicates that $\mathrm{C} 3$ is not likely to inhibit cell migration.

\section{Conclusion}

An automated image processing framework for cell migration analysis is proposed in this paper. The main contribution is an approach to estimate the location of the ROI, followed by segmentation of individual cells within the ROI. In order to evaluate the approach, the ROI estimation and cell segmentation are examined by biologists. The results of the proposed image processing approach proved to be comparable to human raters, which verifies that the automatic image processing framework is a useful tool to quantify cell migration.

\section{References}

1. Zimmer B, Lee G, Balmer NV, et al. Evaluation of developmental toxicants and signaling pathways in a functional test based on the migration of human neural crest cells. Environ Health Perspect. 2012;120(8):1116-22.

2. Riess T, Dietz C, Tomas M, et al. Automated Image Processing for the Analysis of DNA Repair Dynamics. In: Proc. 3rd International Conference on Bioinformatics, Biocomputational Systems and Biotechnologies (BioTechno); 2011. p. 31-6.

3. Berthold MR, Cebron N, Dill F, et al. KNIME: The Konstanz Information Miner. In: Proc. Data Analysis, Machine Learning and Applications; 2008. p. 319-26.

4. Jones TR, Carpenter AE, Golland P. Voronoi-Based Segmentation of Cells on Image Manifolds. In: Proc. Computer Vision for Biomedical Image Applications (CVBIA); 2005. p. $535-43$. 